\title{
On the Additive Constant of the $k$-server Work Function Algorithm
}

\author{
Yuval Emek* $\quad$ Pierre Fraigniaud ${ }^{\dagger} \quad$ Amos Korman $^{\ddagger} \quad$ Adi Rosén ${ }^{\S}$
}

\begin{abstract}
We consider the Work Function Algorithm for the $k$-server problem $[2,3]$. We show that if the Work Function Algorithm is c-competitive, then it is also strictly (2c)-competitive. As a consequence of [3] this also shows that the Work Function Algorithm is strictly $(4 k-2)$ competitive.
\end{abstract}

\section{Introduction}

A (deterministic) online algorithm $\mathrm{Alg}$ is said to be $c$-competitive if for all finite request sequences $\rho$, it holds that $\operatorname{Alg}(\rho) \leq c \cdot O P T(\rho)+\beta$, where $\operatorname{Alg}(\rho)$ and $O P T(\rho)$ are the costs incurred by $\mathrm{Alg}$ and the optimal algorithm, respectively, on $\sigma$ and $\beta$ is a constant independent of $\rho$. When this condition holds for $\beta=0$, then $\mathrm{Alg}$ is said to be strictly c-competitive.

The $k$-server problem is one of the most extensively studied online problems (cf. [1]). To date, the best known competitive ratio for the $k$-server problem on general metric spaces is $2 k-1$ [3], which is achieved by the Work Function Algorithm [2]. A lower bound of $k$ for any metric space with at least $k+1$ nodes is also known [4]. The question whether online algorithms are strictly competitive, and in particular if there is a strictly competitive $k$-server algorithm, is of interest for two reasons. First, as a purely theoretical question. Second, at times one attempts to build a competitive online algorithm by repeatedly applying another online algorithm as a subroutine. In that case, if the online algorithm applied as a subroutine is not strictly competitive, the resulting online algorithm may not be competitive at all due to the growth of the additive constant with the length of the request sequence.

${ }^{*}$ Tel Aviv University, Tel Aviv, 69978 Israel. E-mail: yuvale@eng.tau.ac.il. This work was partially done during this author's visit at LIAFA, CNRS and University Paris Diderot, supported by Action COST 295 DYNAMO.

${ }^{\dagger}$ CNRS and University Paris Diderot, France. Email: pierre.fraigniaud@liafa.jussieu.fr. Additional support from the ANR project ALADDIN, by the INRIA project GANG, and by COST Action 295 DYNAMO.

${ }^{\ddagger}$ CNRS and University Paris Diderot, France. Email: amos.korman@liafa.jussieu.fr. Additional support from the ANR project ALADDIN, by the INRIA project GANG, and by COST Action 295 DYNAMO.

${ }^{\S}$ CNRS and University of Paris 11, France. Email: adiro@lri.fr. Research partially supported by ANR projects AlgoQP and ALADDIN. 
In this paper we show that there exists a strictly competitive $k$-server algorithm for general metric spaces. In fact, we show that if the Work Function Algorithm is $c$-competitive, then it is also strictly (2c)-competitive. As a consequence of [3], we thus also show that the Work Function Algorithm is strictly $(4 k-2)$-competitive.

\section{Preliminaries}

Let $\mathcal{M}=(V, \delta)$ be a metric space. We consider instances of the $k$-server problem on $\mathcal{M}$, and when clear from the context, omit the mention of the metric space. At any given time, each server resides in some node $v \in V$. A subset $X \subseteq V,|X|=k$, where the servers reside is called a configuration. The distance between two configurations $X$ and $Y$, denoted by $D(X, Y)$, is defined as the weight of a minimum weight matching between $X$ and $Y$. In every round, a new request $r \in V$ is presented and should be served by ensuring that a server resides on the request $r$. The servers can move from node to node, and the movement of a server from node $x$ to node $y$ incurs a cost of $\delta(x, y)$.

Fix some initial configuration $A_{0}$ and some finite request sequence $\rho$. The work function $w_{\rho}(X)$ of the configuration $X$ with respect to $\rho$ is the optimal cost of serving $\rho$ starting in $A_{0}$ and ending up in configuration $X$. The collection of work function values $w_{\rho}(\cdot)=\left\{\left(X, w_{\rho}(X)\right)|X \subseteq V| X \mid,=k\right\}$ is referred to as the work vector of $\rho$ (and initial configuration $A_{0}$ ).

A move of some server from node $x$ to node $y$ in round $t$ is called forced if a request was presented at $y$ in round $t$. (An empty move, in case that $x=y$, is also considered to be forced.) An algorithm for the $k$-server problem is said to be lazy if it only makes forced moves. Given some configuration $X$, an offline algorithm for the $k$-server problem is said to be $X$-lazy if in every round other than the last round, it only makes forced moves, while in the last round, it makes a forced move and it is also allowed to move servers to nodes in $X$ from nodes not in $X$. Since unforced moves can always be postponed, it follows that $w_{\rho}(X)$ can be realized by an $X$-lazy (offline) algorithm for every choice of configuration $X$.

Given an initial configuration $A_{0}$ and a request sequence $\rho$, we denote the total cost paid by an online algorithm $\mathrm{Alg}$ for serving $\rho$ (in an online fashion) when it starts in $A_{0}$ by $\operatorname{Alg}\left(A_{0}, \rho\right)$. The optimal cost for serving $\rho$ starting in $A_{0}$ is denoted by $\operatorname{Opt}\left(A_{0}, \rho\right)=\min _{X}\left\{w_{\rho}(X)\right\}$. The optimal cost for serving $\rho$ starting in $A_{0}$ and ending in configuration $X$ is denoted by $\operatorname{Opt}\left(A_{0}, \rho, X\right)=w_{\rho}(X)$. (This seemingly redundant notation is found useful hereafter.)

Consider some metric space $\mathcal{M}$. In the context of the $k$-server problem, an algorithm Alg is said to be competitive if for any initial configuration $A_{0}$, and any finite request sequence $\rho$, $\operatorname{Alg}\left(A_{0}, \rho\right) \leq c \cdot \operatorname{Opt}\left(A_{0}, \rho\right)+\beta$, where $\beta$ may depend on the initial configuration $A_{0}$, but not on the request sequence $\rho$. Alg is said to be strictly c-competitive if it is $c$-competitive with additive constant $\beta=0$, that is, if for any initial configuration $A_{0}$ and any finite request sequence $\rho$, $\operatorname{Alg}\left(A_{0}, \rho\right) \leq c \cdot \operatorname{Opt}\left(A_{0}, \rho\right)$. As common in other works, we assume that the online algorithm and 
the optimal algorithm have the same initial configuration.

\section{Strictly competitive analysis}

We prove the following theorem.

Theorem 3.1. If the Work Function Algorithm is c-competitive, then it is also strictly (2c)competitive.

In fact, we shall prove Theorem 3.1 for a (somewhat) larger class of $k$-server online algorithms, referred to as robust algorithms (this class will be defined soon). We say that an online algorithm for the $k$-server problem is request-sequence-oblivious, if for every initial configuration $A_{0}$, request sequence $\rho$, current configuration $X$, and request $r$, the action of the algorithm on $r$ after it served $\rho$ (starting in $A_{0}$ ) is fully determined by $X, r$, and the work vector $w_{\rho}(\cdot)$. In other words, a request-sequence-oblivious online algorithm can replace the explicit knowledge of $A_{0}$ and $\rho$ with the knowledge of $w_{\rho}(\cdot)$. An online algorithm is said to be robust if it is lazy, request-sequence-oblivious, and its behavior does not change if one adds to all entries of the work vector any given value $d$. We prove that if a robust algorithm is $c$-competitive, then it is also strictly $(2 c)$-competitive. Theorem 3.1 follows as the work function algorithm is robust.

In what follows, we consider a robust online algorithm Alg and a lazy optimal (offline) algorithm Opt for the $k$-server problem. (In some cases, Opt will be assumed to be $X$-lazy for some configuration $X$. This will be explicitly stated.) We also consider some underlying metric $\mathcal{M}=(V, \delta)$ that we do not explicitly specify. Suppose that Alg is $\alpha$-competitive and given the initial configuration $A_{0}$, let $\beta=\beta\left(A_{0}\right)$ be the additive constant in the performance guarantee.

Subsequently, we fix some arbitrary initial configuration $A_{0}$ and request sequence $\rho$. We have to prove that $\operatorname{Alg}\left(A_{0}, \rho\right) \leq 2 \alpha \operatorname{ppt}\left(A_{0}, \rho\right)$. A key ingredient in our proof is a designated request sequence $\sigma$ referred to as the anchor of $A_{0}$ and $\rho$. Let $\ell=\min \left\{\delta(x, y) \mid x, y \in A_{0}, x \neq y\right\}$. Given that $A_{0}=\left\{x_{1}, \ldots, x_{k}\right\}$, the anchor is defined to be

$$
\sigma=\left(x_{1} \cdots x_{k}\right)^{m}, \text { where } m=\left\lceil\max \left\{\frac{2 k 0 \mathrm{pt}\left(A_{0}, \rho\right)}{\ell}+k^{2}, \frac{2 \alpha 0 \mathrm{pt}\left(A_{0}, \rho\right)+\beta\left(A_{0}\right)}{\ell}\right\}\right]+1 .
$$

That is, the anchor consists of $m$ cycles of requests presented at the nodes of $A_{0}$ in a round-robin fashion.

Informally, we shall append $\sigma$ to $\rho$ in order to ensure that both Alg and Opt return to the initial configuration $A_{0}$. This will allow us to analyze request sequences of the form $(\rho \sigma)^{q}$ as $q$ disjoint executions on the request sequence $\rho \sigma$, thus preventing any possibility to "hide" an additive constant in the performance guarantee of $\operatorname{Alg}\left(A_{0}, \rho\right)$. Before we can analyze this phenomenon, we have to establish some preliminary properties.

Proposition 3.2. For every initial configuration $A_{0}$ and request sequence $\rho$, we have $\operatorname{Opt}\left(A_{0}, \rho, A_{0}\right) \leq 2 \cdot \operatorname{Opt}\left(A_{0}, \rho\right)$. 
Proof. Consider an execution $\eta$ that (i) starts in configuration $A_{0}$; (ii) serves $\rho$ optimally; and (iii) moves (optimally) to configuration $A_{0}$ at the end of round $|\rho|$. The cost of step (iii) cannot exceed that of step (ii) as we can always retrace the moves $\eta$ did in step (ii) back to the initial configuration $A_{0}$. The assertion follows since $\eta$ is a candidate to realize $\operatorname{Opt}\left(A_{0}, \rho, A_{0}\right)$.

Since no moves are needed in order to serve the anchor $\sigma$ from configuration $A_{0}$, it follows that

$$
\operatorname{Opt}\left(A_{0}, \rho\right) \leq \operatorname{Opt}\left(A_{0}, \rho \sigma\right) \leq 2 \cdot \operatorname{Opt}\left(A_{0}, \rho\right)
$$

Proposition 3.2 is also employed to establish the following lemma.

Lemma 3.3. Given some configuration $X$, consider an $X$-lazy execution $\eta$ that realizes $\operatorname{Opt}\left(A_{0}, \rho \sigma, X\right)$. Then $\eta$ must be in configuration $A_{0}$ at the end of round $t$ for some $|\rho| \leq t<|\rho \sigma|$.

Proof. Assume by way of contradiction that $\eta$ 's configuration at the end of round $t$ differs from $A_{0}$ for every $|\rho| \leq t<|\rho \sigma|$. The cost $\operatorname{Opt}\left(A_{0}, \rho \sigma, X\right)$ paid by $\eta$ is at most $2 \cdot \operatorname{Opt}\left(A_{0}, \rho\right)+D\left(A_{0}, X\right)$ as Proposition 3.2 guarantees that this is the total cost paid by an execution that (i) realizes $\operatorname{Opt}\left(A_{0}, \rho, A_{0}\right)$; (ii) stays in configuration $A_{0}$ until (including) round $|\rho \sigma|$; and (iii) moves (optimally) to configuration $X$.

Let $Y$ be the configuration of $\eta$ at the end of round $|\rho|$. We can rewrite the total cost paid by $\eta$ as $\operatorname{Opt}\left(A_{0}, \rho \sigma, X\right)=\operatorname{Opt}\left(A_{0}, \rho, Y\right)+\operatorname{Opt}(Y, \sigma, X)$. Clearly, the former term $\operatorname{Opt}\left(A_{0}, \rho, Y\right)$ is not smaller than $D\left(A_{0}, Y\right)$ which lower bounds the cost paid by any execution that starts in configuration $A_{0}$ and ends in configuration $Y$. We will soon prove (under the assumption that $\eta$ 's configuration at the end of round $t$ differs from $A_{0}$ for every $\left.|\rho| \leq t<|\rho \sigma|\right)$ that the latter term $\operatorname{Opt}(Y, \sigma, X)$ is (strictly) greater than 2.0pt $\left(A_{0}, \rho\right)+D(Y, X)$. Therefore $D\left(A_{0}, Y\right)+2 \cdot 0 \mathrm{pt}\left(A_{0}, \rho\right)+D(Y, X)<\operatorname{Opt}\left(A_{0}, \rho, Y\right)+$ $\operatorname{Opt}(Y, \sigma, X)=\operatorname{Opt}\left(A_{0}, \rho \sigma, X\right)$. The inequality $\operatorname{Opt}\left(A_{0}, \rho \sigma, X\right) \leq 2 \cdot \operatorname{Opt}\left(A_{0}, \rho\right)+D\left(A_{0}, X\right)$ then implies that $D\left(A_{0}, X\right)>D\left(A_{0}, y\right)+D(Y, X)$, in contradiction to the triangle inequality.

It remains to prove that $\operatorname{Opt}(Y, \sigma, X)>2 \cdot \operatorname{Opt}\left(A_{0}, \rho\right)+D(Y, X)$. For that purpose, we consider the suffix $\phi$ of $\eta$ which corresponds to the execution on the subsequence $\sigma$ ( $\phi$ is an $X$-lazy execution that realizes $\operatorname{Opt}(Y, \sigma, X))$. Clearly, $\phi$ must shift from configuration $Y$ to configuration $X$, paying cost of at least $D(Y, X)$. Moreover, since $\phi$ is $X$-lazy, and by the assumption that $\phi$ does not reside in configuration $A_{0}$, it follows that in each of the $m$ cycles of the round-robin, at least one server must move between two different nodes in $A_{0}$. (To see this, recall that each server's move of the lazy execution ends up in a node of $A_{0}$. On the other hand, all $k$ servers never reside in configuration $A_{0}$.) Thus $\phi$ pays a cost of at least $\ell$ per cycle, and $m \ell$ altogether. A portion of this $m \ell$ cost can be charged on the shift from configuration $Y$ to configuration $X$, but we show that the remaining cost is strictly greater than $2 \cdot \operatorname{Opt}\left(A_{0}, \rho\right)$, thus deriving the desired inequality $\operatorname{Opt}(Y, \sigma, X)>2 \cdot \operatorname{Opt}\left(A_{0}, \rho\right)+D(Y, X)$.

The $k$ servers make at least $m$ moves between two different nodes in $A_{0}$ when $\phi$ serves the subsequence $\sigma$, hence there exists some server $s$ that makes at least $m / k$ such moves as part of 
$\phi$. The total cost paid by all other servers in $\phi$ is bounded from below by their contribution to $D(Y, X)$. As there are $k$ nodes in $A_{0}$, at most $k$ out of the $m / k$ moves made by $s$ arrive at a new node, i.e., a node which was not previously reached by $s$ in $\phi$. Therefore at least $m / k-k$ moves of $s$ cannot be charged on its shift from $Y$ to $X$. It follows that the cost paid by $s$ in $\phi$ is at least $(m / k-k) \ell$ plus the contribution of $s$ to $D(Y, X)$. The assertion now follows by the definition of $m$, since $(m / k-k) \ell>2 \cdot \operatorname{Opt}\left(A_{0}, \rho\right)$.

Since the optimal algorithm Opt is assumed to be lazy, Lemma 3.3 implies the following corollary. Corollary 3.4. If the optimal algorithm Opt serves a request sequence of the form $\rho \sigma \tau$ (for any choice of suffix $\tau$ ) starting from the initial configuration $A_{0}$, then at the end of round $|\rho \sigma|$ it must be in configuration $A_{0}$.

Consider an arbitrary configuration $X$. We want to prove that $w_{\rho \sigma}(X) \geq w_{\rho \sigma}\left(A_{0}\right)+D\left(A_{0}, X\right)$. To this end, assume by way of contradiction that $w_{\rho \sigma}(X)<w_{\rho \sigma}\left(A_{0}\right)+D\left(A_{0}, X\right)$. Fix $w_{0}=w_{\rho \sigma}\left(A_{0}\right)$. Lemma 3.3 guarantees that an $X$-lazy execution $\eta$ that realizes $w_{\rho \sigma}(X)=\operatorname{Opt}\left(A_{0}, \rho \sigma, X\right)$ must be in configuration $A_{0}$ at the end of some round $|\rho| \leq t<|\rho \sigma|$. Let $w_{t}$ be the cost paid by $\eta$ up to the end of round $t$. The cost paid by $\eta$ in order to move from $A_{0}$ to $X$ is at least $D\left(A_{0}, X\right)$, hence $w_{\rho \sigma}(X) \geq w_{t}+D\left(A_{0}, X\right)$. Therefore $w_{t}<w_{0}$, which derives a contradiction, since $w_{0}$ can be realized by an execution that reaches $A_{0}$ at the end of round $t$ and stays in $A_{0}$ until it completes serving $\sigma$ without paying any more cost. As $w_{\rho \sigma}(X) \leq w_{\rho \sigma}\left(A_{0}\right)+D\left(A_{0}, X\right)$, we can establish the following corollary.

Corollary 3.5. For every configuration $X$, we have $w_{\rho \sigma}(X)=w_{\rho \sigma}\left(A_{0}\right)+D\left(A_{0}, X\right)$.

Recall that we have fixed the initial configuration $A_{0}$ and the request sequence $\rho$ and that $\sigma$ is their anchor. We now turn to analyze the request sequence $\chi=(\rho \sigma)^{q}$, where $q$ is a sufficiently large integer that will be determined soon. Corollary 3.4 guarantees that Opt is in the initial configuration $A_{0}$ at the end of round $|\rho \sigma|$. By induction on $i$, it follows that Opt is in $A_{0}$ at the end of round $i \cdot|\rho \sigma|$ for every $1 \leq i \leq q$. Therefore the total cost paid by Opt on $\chi$ is merely

$$
\operatorname{Opt}\left(A_{0}, \chi\right)=q \cdot \operatorname{Opt}\left(A_{0}, \rho \sigma\right) \text {. }
$$

Suppose by way of contradiction that the online algorithm Alg, when invoked on the request sequence $\rho \sigma$ from initial configuration $A_{0}$, does not end up in $A_{0}$. Since Alg is lazy, we conclude that Alg is not in configuration $A_{0}$ at the end of round $t$ for any $|\rho| \leq t<|\rho \sigma|$. Therefore in each cycle of the round-robin, Alg moves at least once between two different nodes in $A_{0}$, paying cost of at least $\ell$. By the definition of $m$ (the number of cycles), this sums up to $\operatorname{Alg}\left(A_{0}, \rho \sigma\right) \geq m \ell>$ $2 \alpha 0 \operatorname{pt}\left(A_{0}, \rho\right)+\beta\left(A_{0}\right)$. By inequality (1), we conclude that $\operatorname{Alg}\left(A_{0}, \rho \sigma\right)>\alpha 0 \operatorname{pt}\left(A_{0}, \rho \sigma\right)+\beta\left(A_{0}\right)$, in contradiction to the performance guarantee of Alg. It follows that $\mathrm{Alg}$ returns to the initial configuration $A_{0}$ after serving the request sequence $\rho \sigma$.

Consider some two request sequences $\tau$ and $\tau^{\prime}$. We say that the work vector $w_{\tau}(\cdot)$ is $d$-equivalent to the work vector $w_{\tau^{\prime}}(\cdot)$, where $d$ is some real, if $w_{\tau}(X)-w_{\tau^{\prime}}(X)=d$ for every $X \subseteq V,|X|=k$. It 
is easy to verify that if $w_{\tau}(\cdot)$ is $d$-equivalent to $w_{\tau^{\prime}}(\cdot)$, then $w_{\tau r}(\cdot)$ is $d$-equivalent to $w_{\tau^{\prime} r}(\cdot)$ for any choice of request $r \in V$. Corollary 3.5 guarantees that the work vector $w_{\rho \sigma}(\cdot)$ is $d$-equivalent to the work vector $w_{\omega}(\cdot)$ for some real $d$, where $\omega$ stands for the empty request sequence. (In fact, $d$ is exactly $w_{\rho \sigma}\left(A_{0}\right)$.) By induction on $j$, we show that for every prefix $\pi$ of $\rho \sigma$ and for every $1 \leq i<q$ such that $\left|(\rho \sigma)^{i} \pi\right|=j$, the work vector $w_{(\rho \sigma)^{i} \pi}(\cdot)$ is $d$-equivalent to the work vector $w_{\pi}(\cdot)$ for some real $d$. Therefore the behavior of the robust online algorithm Alg on $\chi$ is merely a repetition $(q$ times) of its behavior on $\rho \sigma$ and

$$
\operatorname{Alg}\left(A_{0}, \chi\right)=q \cdot \operatorname{Alg}\left(A_{0}, \rho \sigma\right)
$$

We are now ready to establish the following inequality:

$$
\begin{aligned}
\operatorname{Alg}\left(A_{0}, \rho\right) & \leq \operatorname{Alg}\left(A_{0}, \rho \sigma\right) \\
& =\frac{\operatorname{Alg}\left(A_{0}, \chi\right)}{q} \quad \text { by inequality }(3) \\
& \leq \frac{\alpha \operatorname{pt}\left(A_{0}, \chi\right)+\beta\left(A_{0}\right)}{q} \quad \text { by the performance guarantee of } \mathrm{Alg} \\
& =\frac{\alpha q \operatorname{pt}\left(A_{0}, \rho \sigma\right)+\beta\left(A_{0}\right)}{q} \quad \text { by inequality }(2) \\
& \leq \frac{2 \alpha q \operatorname{pt}\left(A_{0}, \rho\right)+\beta\left(A_{0}\right)}{q} \quad \text { by inequality }(1) \\
& =2 \alpha 0 \operatorname{pt}\left(A_{0}, \rho\right)+\frac{\beta\left(A_{0}\right)}{q} .
\end{aligned}
$$

For any real $\epsilon>0$, we can fix $q=\left\lceil\beta\left(A_{0}\right) / \epsilon\right\rceil+1$ and conclude that $\operatorname{Alg}\left(A_{0}, \rho\right)<2 \alpha \operatorname{ppt}\left(A_{0}, \rho\right)+\epsilon$. Theorem 3.1 follows.

As the Work Function Algorithm is known to be $(2 k-1)$-competitive [3], we also get the following corollary.

Corollary 3.6. The Work Function Algorithm is strictly $(4 k-2)$-competitive.

Acknowledgments We thank Elias Koutsoupias for useful discussions.

\section{References}

[1] A. Borodin and R. El-Yaniv, Online Computation and Competitive Analysis. Cambridge University Press, 1998.

[2] M. Chrobak and L.L. Larmore. The server problem and on-line games. In On-line algorithms: Proc. of a DIMACS Workshop. DIMACS Series in Discrete Mathematics and Theoretical Computer Science, vol. 7, pages 11-64, 1991. 
[3] E. Koutsoupias and C.H. Papadimitriou. On the $k$-server conjecture. J. $A C M, 42(5): 971-983$, 1995.

[4] M.S. Manasse, L.A. McGeoch, and D.D. Sleator. Competitive algorithms for server problems. Journal of Algorithms, 11:208-230, 1990. 